\title{
Endovascular Treatment of Intracranial Anterior Circulation Aneurysms with Flow Diverters: A Single Centre Experience with Mid- and Long-Term Results
}

\author{
Ezel YALTIRIK BILGIN ${ }^{1}$, Baran ONAL ${ }^{1}$, Hakan EMMEZ ${ }^{2}$, Koray AKKAN ${ }^{1}$, Erhan ILGIT ${ }^{1}$, Erkan BILGIN ${ }^{1}$, \\ Emetullah CINDIL ${ }^{1}$, Alp Ozgun BORCEK ${ }^{2}$
}

${ }^{1}$ Gazi University, School of Medicine, Department of Radiology, Ankara, Turkey

${ }^{2}$ Gazi University, School of Medicine, Department of Neurosurgery, Ankara, Turkey

\section{ABSTRACT}

AIM: We report our experience with flow diverter devices in the treatment of intracranial aneurysms arising from the anterior circulation with mid- and long-term follow-up.

MATERIAL and METHODS: Seventy-eight aneurysms in 61 patients (range 25-81 years, 13 male, 48 female) were treated with flow diverter devices. Forty (51.3\%) aneurysms were treated with a pipeline embolization device (PED), 24 (30.8\%) aneurysms were treated with a SILK stent, 12 (15.4\%) aneurysms were treated with a flow redirection endoluminal device (FRED) and two aneurysms were treated with a P64 Flow Modulation Device (P64). Angiographic follow-up data at six months and one year were recorded and the occlusion degrees of the aneurysms were evaluated according to the scale developed by Kamran et al.

RESULTS: At six-month follow-up, the complete occlusion (grade 4) rate was $60.8 \%$ and at one-year this rate had increased to $74.3 \%$. Statistical analysis revealed a significant difference $(p=0.002)$ between six-month and one-year follow-up results but there was no significant association $(p=0.531, p=1.000)$ between aneurysm occlusion rate and aneurysm diameter. Two patients $(3.2 \%)$ died due to hemorrhagic complications.

CONCLUSION: Endovascular treatment of intracranial anterior circulation aneurysms with flow diverters is a safe and effective treatment option. A high rate of stable occlusion is achieved at long-term follow-up.

KEYWORDS: Aneurysm, Endovascular treatment, Flow diverter, Stenting

\section{INTRODUCTION}

I

ntracranial aneurysm is a cerebrovascular disease with a prevalence of $0.5 \%$ to $6.0 \%$ (6). It is frequently asymptomatic; however, it may rupture and cause subarachnoid hemorrhage (SAH). Eighty-five percent of intracranial aneurysms originate from the anterior circulation (19).

The aim of intracranial aneurysm treatment is to eliminate the risk of spontaneous rupture and to remove the mass effect of aneurysm. In cases of ruptured aneurysms, the primary aim of the treatment is to prevent recurrent bleeding. Various studies have reported that the risk of recurrent bleeding after SAH is $40 \%$, and the mortality rate reaches $80 \%$ within the first month (5).

Flow diverter devices are densely-braided stents used to decrease blood flow into the intracranial aneurysm. These devices divert blood flow away from the aneurysm, decrease intraaneurysmal flow, trigger progressive thrombosis in the aneurysm, and re-model the parent artery (22). 
Previously used devices have been designed to render and support endosaccular embolization of the aneurysm; however, flow diverter devices have been designed to hemodynamically separate the aneurysm from the parent artery. They provide a skeleton for neointimal formation, which is required for reconstruction of the neck defect.

In contrast to covered stents, spaces existing in the flow diverting systems permit the required amount of blood to flow along the parent artery from which the aneurysm originates, so that both large vessel branches and also small perforating arteries will remain patent (11).

Flow diverter devices are especially indicated in fusiform, large and giant, wide-necked intracranial aneurysms, in small aneurysms that are not appropriate for endovascular coil embolization treatment, in aneurysms with branching parent artery or perforating arteries, and in other intracranial aneurysms that show recurrent aneurysmal filling after the treatment (14). Double antiplatelet therapy is required for the patency of flow diverting systems as they are endoluminal devices. These devices are therefore accepted to be relatively contraindicated in subarachnoid hemorrhage.

In one study, aneurysm occlusion was observed to exist in three phases after treatment with flow diverter devices: in the first phase (1 to 3 months) there is moderate to severe decrease in filling of the aneurysm, in the second phase ( 3 to 12 months) funnel-shaped filling in the neck of the aneurysm, and in the third phase (6 to 18 months) total occlusion. The duration of total occlusion may be prolonged depending on the double antiplatelet therapy and its duration (21).

The present study aimed to report our experience with flow diverters in the treatment of intracranial aneurysms arising from the anterior circulation, with mid- and long-term followup.

\section{- MATERIAL and METHODS}

This study includes the cases of large, giant and wide necked aneurysms or aneurysms in which vessel branches originate from the sac and are not suitable for treatment methods other than flow diverter devices. The criteria for the included aneurysms were those defined in the systematic review by Leung et al. (10). Patients were informed in detail by a member of the research team about the standard treatment by coil embolization, treatment by flow diverter devices, and the benefits and complications of the treatment; informed consent was then obtained from the patients. This study was conducted under the approval of the Institutional Review Board.

At least seven days before the procedure, patients received a $300-600 \mathrm{mg}$ loading dose of clopidogrel, and then $75 \mathrm{mg}$ of clopidogrel orally each day until the procedure. Clopidogrel resistance of each patient was also evaluated by the 'Rapid Platelet Function Assay' (RPFA-ASA) test prior to the procedure. After treatment, dual antiplatelet regimen was continued as $75 \mathrm{mg} /$ day clopidogrel orally for 12 months and aspirin $100 \mathrm{mg} /$ day for life.
During the procedure, all patients were administered heparin intravenous (IV), so that activated clotting time (ACT) could be kept 2 to 2.5 times above the basal level. The heparin infusion was continued for 24 hours after the procedure. Patients with large or giant aneurysms received $8 \mathrm{mg}$ dexamethasone IV immediately after the procedure as an initial dose, continued as $16 \mathrm{mg} /$ day orally for a week, and then tapered and discontinued.

Endovascular treatment with flow diverter devices was performed under general anesthesia. In all patients, a $6 \mathrm{~F}$ $90 \mathrm{~cm}$ long introducer sheath was placed in the distal common carotid artery, a $6 \mathrm{~F}$ distal access guiding catheter was advanced as distally as possible in the internal carotid artery. A 2.4F-2.7F microcatheter was used, with different microguidewires as necessary, to selectively catheterize the distal branch. Flow diverter device deployment was performed under fluoroscopy using the roadmap function in preselected positions. Digital subtraction angiography (DSA) images including 3D rotational angiographies were also obtained before, during and after the procedure.

Follow-up angiographies were planned in the sixth month, first year, and then annually. During the controls, neurological examinations of the patients were repeated and medications were controlled. The aneurysm occlusion was evaluated according to the scale developed by Kamran et al. (8) (Table I). If the aneurysm involved vessels branching from it, the patency of these vessels and of the parent artery was also evaluated.

Data were analyzed by using the SPSS 18.0 statistics program. Descriptive statistics were expressed as percent distribution, mean, median, and minimum and maximum values. In the comparison of two independent groups with two variables, the chi-square test and Fisher's exact test were used. In the comparison of two dependent groups with two variables, the McNemar-Bowker test was used. A type 1 error level less than $5 \%$ was accepted to be significant statistically.

\section{RESULTS}

Seventy-eight anterior circulation aneurysms of 61 patients were treated by using a flow diverter. The mean age of the patients was 51.7 years $(\min =20, \max =81$ years). Females made up $78.6 \%$ of the patients. Locations of the treated anterior circulation aneurysms are listed in Table II.

Of the aneurysms, 50 were small $(<10 \mathrm{~mm}), 17$ were large (10-25 mm), 8 were giant $(>25 \mathrm{~mm})$, and 3 were fusiform. $40(51.3 \%)$ of the aneurysms were treated with a pipeline embolization device (PED), 24 (30.8\%) with a SILK stent, 12 (15.4\%) with a flow redirection endoluminal device (FRED), and two $(2.5 \%)$ with a P64 Flow Modulation Device (P64). In four of the 24 aneurysms treated with SILK stents, treatment was performed with two overlapping stents due to insufficient coverage with only one. A similar technique was used in three of the 40 aneurysms treated with PED. Eighty-five flow diverter devices were implanted in a total of 78 aneurysms. 
Two of the 61 patients died from a hemorrhagic event due to flow diverter device treatment. Except these cases, no major or minor intraprocedural complications developed in this series. Another patient died due to iatrogenic diffuse subcutaneous

Table I: Grading Schema of Aneurysmal Occlusion After the Treatment (8)

\begin{tabular}{ll}
\hline Grade & Aneurysmal Filling \\
\hline 0 & No difference in aneurysmal filling \\
\hline 1 & $\begin{array}{l}\text { More than } 50 \% \text { residual filling when compared to } \\
\text { pretreatment state }\end{array}$ \\
\hline 2 & $\begin{array}{l}\text { Less than } 50 \% \text { residual filling when compared to } \\
\text { pretreatment state }\end{array}$ \\
\hline 3 & Residual filling only in the neck of the aneurysm \\
\hline
\end{tabular}

Table II: Locations of the Treated Anterior Circulation Aneurysms

\begin{tabular}{lc}
\hline Location & Number / \% \\
\hline Anterior cerebral artery & $2 /(2.6 \%)$ \\
A2 segment & $2 /(2.6 \%)$ \\
\hline Middle cerebral artery & $17 /(21.8 \%)$ \\
M1 segment & $14 /(18.0 \%)$ \\
MCA bifurcation & $3 /(3.8 \%)$ \\
\hline Internal carotid artery & $59 /(75.6 \%)$ \\
Petrous segment(C2) & $2 /(2.6 \%)$ \\
Cavernous segment (C4) & $38 /(48.6 \%)$ \\
Clinoid segment (C5) & $3 /(3.8 \%)$ \\
Ophthalmic segment (C6) & $8 /(10.3 \%)$ \\
Communicating segment (C7) & $8 /(10.3 \%)$ \\
\hline
\end{tabular}

emphysema following spontaneous pneumothorax in the postoperative second month, unrelated with the procedure. In addition, one patient was excluded from angiographic followup due to total ICA occlusion after the procedure, and was followed by MRI. This patient has been asymptomatic due to patent anterior communicating artery supply.

Except the patients excluded from follow-up, all cases (74 aneurysms) were controlled angiographically at the sixth month and first year. Observations of the aneurysms at the sixth month were as follows: complete occlusion (grade 4 occlusion) in $45(60.8 \%)$, grade 3 occlusion in nine $(12.2 \%)$, and grade 2 occlusion in 20 (27\%).

The first year control angiographies were performed in 57 patients (74 aneurysms) and the results were as follows: grade 4 occlusion in 55 (74.3\%), grade 3 occlusion in $12(16.2 \%)$ and grade 2 occlusion in seven $(9.5 \%)$.

Furthermore, occlusion rates in relation to aneurysmal dimensions (small, large, giant) were also determined in the sixth month and the first year angiographic controls. $74 \%$ of the small aneurysms showed grade 3-4 occlusions in the sixth month controls; this value was $66.6 \%$ in the large and giant aneurysms. No correlations were found between the aneurysmal dimensions and occlusion rates $(p=0.531)$ at six months. $90 \%$ of the small aneurysms showed grade 3-4 occlusions, and this value was $90.4 \%$ in the large and giant aneurysms at first year angiographic controls; no correlations were found between the aneurysmal dimensions and occlusion rates $(p=1.000)$ at first year follow-up.

Of the 20 aneurysms that showed grade 2 occlusion at the sixth month angiographic control, $40 \%(n=8)$ showed grade 4 (Figure $1 A-C)$, and $25 \%(n=5)$ showed grade 3 occlusions in the first year control. Of the nine aneurysms that showed grade 3 occlusion in the sixth month, $22 \%(n=2)$ showed grade 4 occlusion in the first year. Differences in occlusion
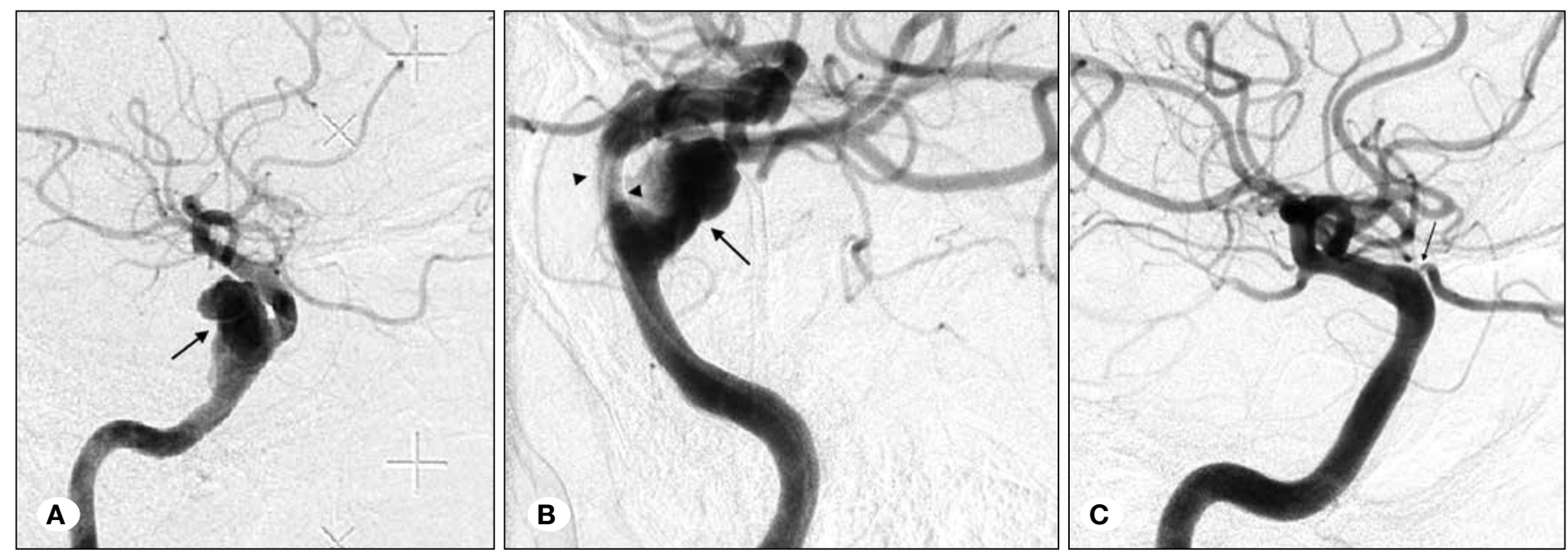

Figure 1: A) Dissecting pseudoaneurysm $15 \mathrm{~mm}$ in size with irregular contours in the precavernous segment of right internal carotid artery is monitored in lateral projection images of DSA (black arrow). B) After placing a $5.0 \times 35$ mm sized PED into the parent artery (arrowheads), significant stagnation of the contrast medium in the aneurysm sac can be identified (arrow). C) One-year control angiogram in lateral projection shows total occlusion (grade 4) of the aneurysm and narrowing of the ophtalmic artery ostium (arrow) which is covered by PED. 
rates determined between the sixth month and the first year controls were statistically significant $(p=0.002)$.

An anterior cerebral artery (ACA) pericallosal artery aneurysm, which includes the frontal branch, was treated with PED; posttreatment control angiographies in the sixth month and the first year showed grade 2 and grade 4 occlusions respectively, and the frontal branch that originates was observed to remain patent (Figure $2 \mathrm{~A}-\mathrm{C}$ ). An internal carotid artery (ICA) aneurysm that branches the posterior communicating artery was treated with PED; in the post-treatment sixth month control angiography, the aneurysm showed grade 2 occlusion, and the calibration of the branching artery decreased. In the first year control, the aneurysm showed grade 4 occlusion, and the posterior communicating artery was observed not to be filled up; however, the right posterior cerebral artery was indicated to be supplied by the posterior circulation (Figure $3 A-C$ ).

Three cavernous segment aneurysms treated with SILK and one treated with FRED showed a stenosis of less than $30 \%$ in the stented segment at the sixth month and one-year followup. The cases that showed stenosis were asymptomatic, and the endovascular approach was therefore not considered.

\section{DISCUSSION}

Treatment of especially wide necked, non-saccular, large, giant, or dissected aneurysms by standard coil embolization
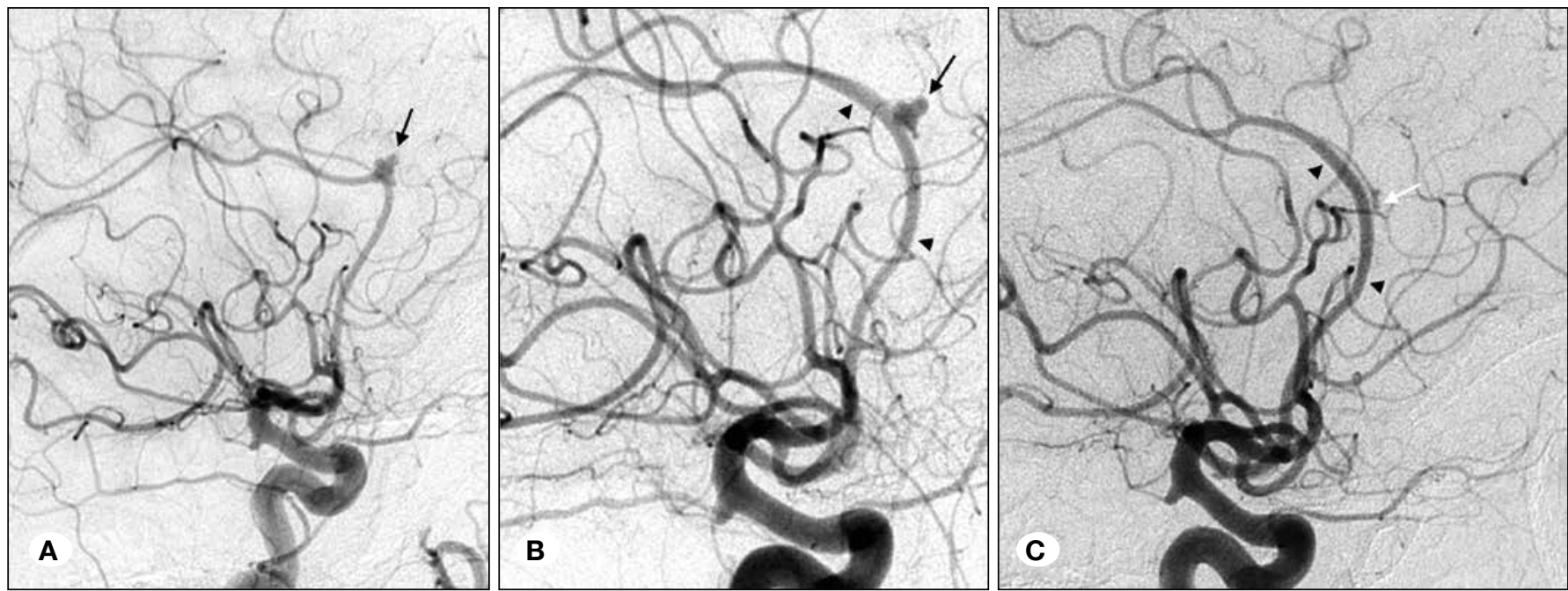

Figure 2: A) A $4 \mathrm{~mm}$ aneurysm with comperatively irregular contours in the pericallosal branch of right anterior cerebral artery and frontal branch originating from the aneurysm sac is monitored in lateral projection DSA (black arrow). B) Six-month control angiogram after treatment with a PED shows grade 2 occlusion of the aneurysm sac (black arrow). Also proximal and distal parts of the PED can be identified (arrowheads). C) One-year control angiogram demonstrates complete (grade 4) occlusion of aneurysm sac and patency of frontal branch and parent artery (white arrow and arrowheads).
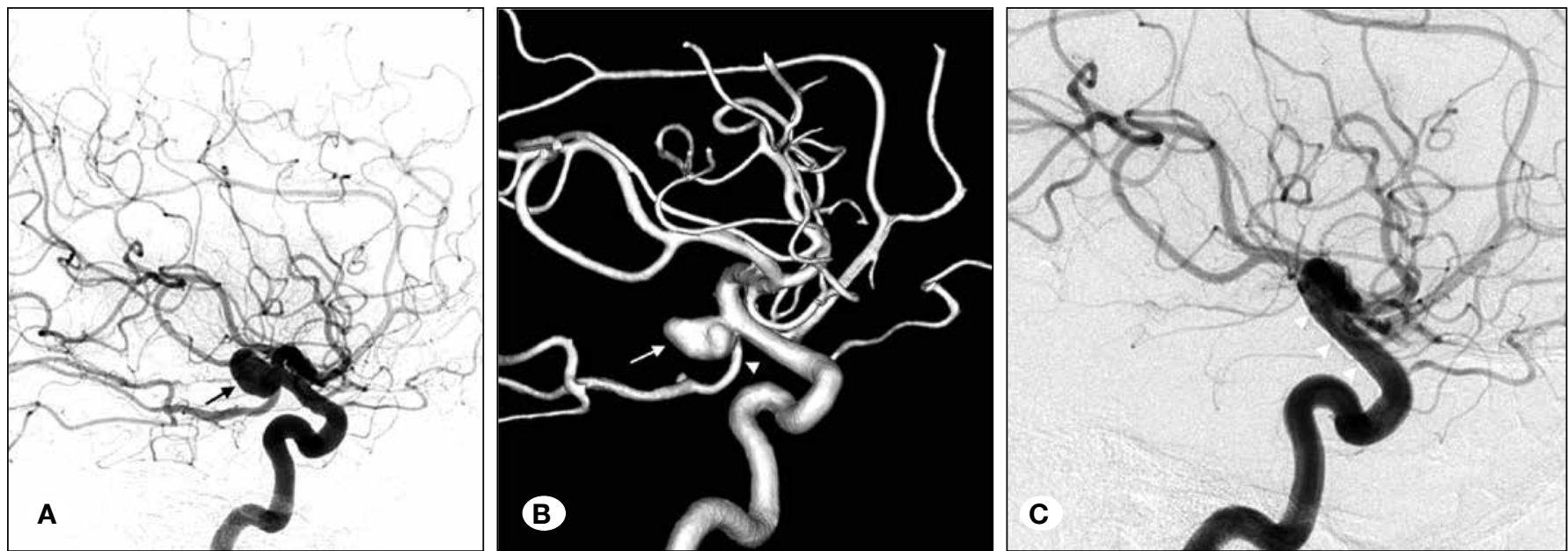

Figure 3: A, B) DSA and 3D lateral projection images shows an internal carotid artery communicating segment aneurysm (black and white arrows) that is arising from the ostium of the posterior communicating artery (arrowhead). C) One-year control angiogram after treatment in lateral projection shows correct posterior wall apposition of the flow diverter (arrowheads) and total (grade 4) occlusion of the aneurysm sac. Note that posterior communicating artery is not filling anymore. 
leads to low success rates, or this treatment cannot be applied due to anatomical issues. In addition, solely coil embolization is not sufficient for the reconstruction of a non-existing wall in wide necked aneurysms.

In many studies, complete occlusion rates were low in the angiographic controls performed after coil embolization treatment. Raymond et al. determined a complete occlusion rate of $38.3 \%$ in the first year control angiographies of 353 aneurysms treated with coil embolization (16). Kole et al. determined a complete occlusion rate of $19 \%$ in the long-term ( $18^{\text {th }}$ month) angiographic controls of 131 aneurysms treated with coil embolization (9). Recurrences of $35 \%$ for large aneurysms, and $59.1 \%$ for giant aneurysms after the treatment with coil embolization in the long-term ( $5^{\text {th }}$ year) angiographic controls were reported (12). After the standard coil treatment, new techniques were developed for the treatment and reconstruction of particularly complex aneurysms (14).

Especially in the coiling of wide necked aneurysms, the technique of modelling the neck by a balloon was developed in order to prevent the coils from hanging through the wide aneurysm neck into the parent artery or from migrating to the lumen, and to provide a perfect stabilization. When a micro-catheter is introduced into the aneurysm, the balloon is inflated to support the coils in the aneurysm, and they are, thus, stabilized. This method is preferred particularly in the treatment of bleeding wide necked aneurysms. Studies have reported that aneurysmal occlusion rates are higher when compared to the standard coil embolization, and thromboembolic complication rates were reported to be similar to those of the standard treatment (18).

The other treatment option is surgical clipping. In a prospective study on 1917 patients with surgical treatment (clipping), the 1-year morbidity rate was $9.8 \%$ and the mortality rate was $2.3 \%$. Surgical success rates decrease with older age and history of previous stroke (20). In the literature, aneurysm recurrence rates after complete clipping vary from $0 \%$ to $2.4 \%(4)$.

Flow diverter devices are densely braided intracranial stents designed to decrease intraaneurysmal blood flow in order to isolate the aneurysm from circulation, and to provide sufficient flow so that vessels branching from the aneurysm and also small perforating arteries will remain patent; they are also used in remodeling the parent artery (21).

Studies have proven the efficacy of treatment with flow diverter devices. The complete occlusion rates of 63 aneurysms treated with PED were 56\%, 93\%, and 95\% in the third, sixth months and first year of post-treatment control angiographies, respectively. The lower complete occlusion rate determined in the third month when compared to the sixth month and first year, was attributed to the antiplatelet therapy applied in order to prevent thrombosis formation in the stent (11).

In a multi-centered study performed on 31 aneurysms, total occlusion was $93 \%$ in the sixth month angiographic controls after PED treatment (13).

The study by Byrne et al. evaluated 68 aneurysms treated with a SILK stent. In the angiographic controls performed just after the procedure, complete occlusion, neck remnant, and aneurysmal residual filling rates were $10 \%, 6 \%$, and $84 \%$, respectively. In the angiographic controls performed one month after the completion of antiplatelet treatment, complete occlusion, neck remnant, and aneurysmal residual filling rates were $49 \%, 26 \%$, and $25 \%$, respectively (2).

In a study by Yavuz et al., which was performed with 25 middle cerebral artery (MCA) aneurysms, the total occlusion rate in the long-term $\left(12^{\text {th }}-18^{\text {th }}\right.$ months $)$ angiographic controls after the PED treatment was $84 \%$ (21).

In the literature, total occlusion rates after the treatment of aneurysms with flow diverters vary from $55 \%$ to $95 \%$. Furthermore, it was indicated that occlusion rates were independent of the aneurysm dimensions, and thus the belief that small aneurysms are occluded at higher rates after endovascular treatment is no longer valid (1).

The degree of aneurysm occlusion has been evaluated according to the scale developed by Kamran et al.(8). Recently, Cekirge et al. proposed a new classification for intracranial aneurysms treated by endovascular means, including coiling with or without adjunctive devices, intrasaccular flow modifiers, flow diversion or any combination (3).

In the current study, post-treatment angiographic controls in the sixth month were performed in 74 anterior circulation aneurysms, and occlusion findings were as follows: grade 4 in $60.8 \%$, grade 3 in $12.2 \%$, and grade 2 in $27 \%$. The first year control angiographies were also applied to 74 aneurysms, and occlusion findings were as follows: grade 4 in $74.3 \%$, grade 3 in $16.2 \%$, and grade 2 in $9.5 \%$. The difference between the sixth month and first year occlusion rates was statistically significant $(p=0.002)$. Complete occlusion rates were considered to be similar to the literature, and we also considered that this rate would increase more in the long-term controls due to cessation of double antiplatelet therapy. The current study also determined that the degree of aneurysmal occlusion is independent of the aneurysmal dimensions.

In endovascular treatment with flow diverter devices, more than one stent may be overlapped or may be placed telescopically, and the scope may thus be increased such as re-treatment in cases of residual filling or re-growth. In the current study, seven aneurysms were treated with this method. Inadequate coverage was the reason for overlapping and no side branches were arising from these aneurysms in our series.

Due to double antiplatelet therapy and the duration of this therapy after endovascular treatment with flow diverter devices, complete occlusion develops during long-term follow up. Therefore long-term controls provide better results in determining the efficacy of treatment.

If aneurysms indicated to be treated with flow diverter devices are treated with a standard endovascular technique, the mortality and morbidity rates increase when compared to flow diverter devices, since the standard method requires more auxiliary equipment.

Intraprocedural and postprocedural complications may develop. These complications may be mostly hemorrhagic 
or thromboembolic events, and mass effect symptoms worsening or continuing after the procedure. Hemorrhagic events may be related to the dual antiplatelet therapy, which is a component of the treatment. Furthermore, early- or late-term aneurysm ruptures have also been reported in the literature. Early-term aneurysm ruptures have been attributed to the hemodynamic changes in the aneurysm wall, and lateterm aneurysm ruptures have been attributed to the autolysis of the thrombosed aneurysm wall.

In a meta-analysis study, in addition to their efficacy of treatment, flow diverter devices were determined to cause non-negligible complication rates, including $7 \%-12 \%$ mortality and $0-7 \%$ morbidity. In this study, both ischemic and hemorrhagic complications seen after treatment of large aneurysms were reported to be at higher rates when compared to the small aneurysms; this result was attributed to the technical difficulties, and to the instability of large lesions (1). In the present study, the mortality rate was 3.2\% (2 patients), and was due to hemorrhagic complications; this rate is lower than those reported in the literature.

In contrast to the covered stents, flow diverter devices are designed to permit the required amount of blood to flow along the parent artery from which the aneurysm originates, so that both large vessel branches and small perforating arteries would remain patent. In a study by Kallmes et al. performed on rabbits, branches extruding from the aneurysm were shown to remain patent in long-term controls (7). Another study also reported ophthalmic artery occlusion without vision loss in $25 \%$ of cases after treatment of paraclinoid artery aneurysms with flow diverter devices (15). This may be explained by the intensive collaterals of external carotid artery branches, which support the ophthalmic artery. Another study reported longterm controls after the treatment of 46 aneurysms that include branches; aneurysms were treated with flow diverter devices. Five aneurysms included the posterior communicating artery extruding from the aneurysm, and this artery was occluded after the treatment. In these cases, the ipsilateral posterior cerebral artery was filled from the posterior circulation. Like the anterior choroidal artery, other arteries extruding from the aneurysm were patent; this shows that blood requiring arteries due to inadequate distal collateral supply, or arteries with adequate pressure gradients, remain patent after treatment (17). In the current study, the frontal branch arising from ACA pericallosal aneurysm which was treated with PED remained patent due to inadequate distal collateral supply in the sixth month and first year post-treatment control angiographies while the aneurysm showed grade 2 and grade 4 occlusion, respectively. In the other case, the posterior communicating artery arising from the ICA aneurysm was occluded in the first year control while the aneurysm showed grade 4 occlusion. The posterior cerebral artery was observed to be supplied by the posterior circulation.

\section{CONCLUSION}

Flow diverter devices provide an efficient and safe method of treatment, especially in fusiform, large, giant, wide necked intracranial aneurysms; small aneurysms that are not appropriate for endovascular coil embolization treatment; aneurysms that give off side branches or perforating arteries; and other intracranial aneurysms that show recurrent aneurysmal filling after the treatment. A high rate of stable occlusion is achieved at long-term follow-up.

\section{REFERENCES}

1. Brinjikji W, Murad MH, Lanzino G, Cloft HJ, Kallmes DF: Endovascular treatment of intracranial aneurysms with flow diverters: A meta-analysis. Stroke 44:442-447, 2013

2. Byrne JV, Beltechi R, Yarnold JA, Birks J, Kamran M: Early experience in the treatment of intra-cranial aneurysms by endovascular flow diversion: A multicentre prospective study. PLoS One 2:5-10, 2010

3. Cekirge HS, Saatci I: A new aneurysm occlusion classification after the impact of flow modification. AJNR 37:19-24, 2016

4. Goksu E, Korkmaz E, Akyuz M, Ozgur O, Sindel T, Tuncer R: The analysis of long-term follow-up screening in patients with surgically treated intracranial aneurysms. Turk Neurosurg 25: 404-409, 2015

5. Hop JW, Rinkel GJ, Algra A, Van Gijn J: Case-fatality rates and functional outcome after subarachnoid hemorrhage: A systematic review. Stroke 28:660-664, 1997

6. Johnston SC, Higashida RT, Barrow DL, Caplan LR, Dion JE, Hademenos G, Hopkins LN, Molyneux A, Rosenwasser RH, Vinuela F, Wilson CB; Committee on Cerebrovascular Imaging of the American Heart Association Council on Cardiovascular Radiology: Recommendations for the endovascular treatment of intracranial aneurysms: A statement for healthcare professionals from the Committee on Cerebrovascular Imaging of the American Heart Association Council on Cardiovascular Radiology. Stroke 33:2536-2544, 2002

7. Kallmes DF, Ding YH, Dai D, Kadirvel R, Lewis DA, Cloft HJ: A new endoluminal, flow-disrupting device for treatment of saccular aneurysms. Stroke 38:2346-2352, 2007

8. Kamran M, Yarnold J, Grunwald IQ, Byrne JV: Assessment of angiographic outcomes after flow diversion treatment of intracranial aneurysms: A new grading schema. Neuroradiology 53: 501-508, 2011

9. Kole MK, Pelz DM, Kalapos P, Lee DH, Gulka IB, Lownie SP: Endovascular coil embolization of intracranial aneurysms: Important factors related to rates and outcomes of incomplete occlusion. J Neurosurg 102:607-615, 2005

10. Leung GK, Tsang AC, Lui WM: Pipeline embolization device for intracranial aneurysm: A systematic review. Clin Neuroradiol 22:295-303, 2012

11. Lylyk P, Miranda C, Ceratto R, Ferrario A, Scrivano E, Luna HR, Berez AL, Tran Q, Nelson PK, Fiorella D: Curative endovascular reconstruction of cerebral aneurysms with the pipeline embolization device: The Buenos Aires experience. Neurosurgery 64: 632-642, 2009

12. Murayama Y, Nien YL, Duckwiler G, Gobin YP, Jahan R, Frazee J, Martin N, Viñuela F: Guglielmi detachable coil embolization of cerebral aneurysms: 11 years' experience. J Neurosurg 98: 959-966, 2003

13. Nelson PK, Lylyk P, Szikora I, Wetzel SG, Wanke I, Fiorella D: The pipeline embolization device for the intracranial treatment of aneurysms trial. AJNR 32:34-40, 2011 
14. Pierot L: Flow diverter stents in the treatment of intracranial aneurysms: Where are we? J Neuroradiol 38:40-46, 2011

15. Puffer RC, Kallmes DF, Cloft HJ, Lanzino G: Patency of the ophthalmic artery after flow diversion treatment of paraclinoid aneurysms. J Neurosurg 116:892-896, 2012

16. Raymond J, Guilbert F, Weill A, Georganos SA, Juravsky L, Lambert A, Lamoureux J, Chagnon M, Roy D: Long-term angiographic recurrences after selective endovascular treatment of aneurysms with detachable coils. Stroke 34: 1398-1403, 2003

17. Saatci I, Yavuz K, Ozer C, Geyik S, Cekirge HS: Treatment of intracranial aneurysms using the pipeline flow-diverter embolization device: A single- centre experience with longterm follow-up results. AJNR 33:1436-1446, 2012

18. Shapiro M, Babb J, Becske T, Nelson PK: Safety and efficacy of adjunctive balloon remodeling during endovascular treatment of intracranial aneurysms: A literature review. AJNR 29: 1777-1781, 2008
19. Vega C, Kwoon JV, Lavine SD: Intracranial aneurysms: Current evidence and clinical practice. Am Fam Physician 66:601608, 2002

20. Wiebers DO, Whisnant JP, Huston J 3rd, Meissner I, Brown RD Jr, Piepgras DG, Forbes GS, Thielen K, Nichols D, O'Fallon WM, Peacock J, Jaeger L, Kassell NF, Kongable-Beckman GL, Torner JC; International Study of Unruptured Intracranial Aneurysms Investigators: Unruptured intracranial aneurysms: Natural history, clinical outcome, and risks of surgical and endovascular treatment. Lancet 362:103-110, 2003

21. Yavuz K, Geyik S, Saatci I, Cekirge HS: Endovascular treatment of middle cerebral artery aneurysms with flow modification with the use of the pipeline embolization device. AJNR 35: 529-535, 2014

22. Zhang Y, Qian Y, Chong W: Investigation of intracranial aneurysm hemodynamics following flow diverter stent treatment. Med Eng Phys 35: 608-615, 2013 